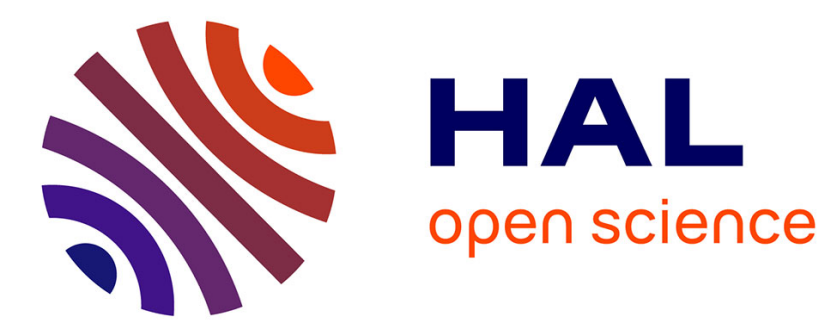

\title{
Changes in phase space during learning an unstable balance
}

\author{
Fabrice Mégrot, Benoit G. Bardy
}

\section{To cite this version:}

Fabrice Mégrot, Benoit G. Bardy. Changes in phase space during learning an unstable balance. Neuroscience Letters, 2006, 402 (1-2), pp.17 - 21. 10.1016/j.neulet.2006.03.041 . hal-01744383

\section{HAL Id: hal-01744383 \\ https://hal-insep.archives-ouvertes.fr/hal-01744383}

Submitted on 27 Mar 2018

HAL is a multi-disciplinary open access archive for the deposit and dissemination of scientific research documents, whether they are published or not. The documents may come from teaching and research institutions in France or abroad, or from public or private research centers.
L'archive ouverte pluridisciplinaire HAL, est destinée au dépôt et à la diffusion de documents scientifiques de niveau recherche, publiés ou non, émanant des établissements d'enseignement et de recherche français ou étrangers, des laboratoires publics ou privés. 


\title{
Changes in phase space during learning an unstable balance
}

\author{
Fabrice Mégrot ${ }^{\mathrm{a}, *}$, Benoît G. Bardy ${ }^{\mathrm{b}, \mathrm{c}}$ \\ ${ }^{a}$ Movement, Action and Performance Laboratory, National Institute of Sports and Physical Education, 11 Tremblay Avenue, 75012 Paris, France \\ ${ }^{\mathrm{b}}$ Institut Universitaire de France, France \\ ${ }^{\mathrm{c}}$ University of Montpellier-1, France
}

Received 8 December 2005; received in revised form 4 March 2006; accepted 17 March 2006

\begin{abstract}
Six participants learned to maintain an unstable balance on a stabilometer, during 6 consecutive days of practice (total of 90 trials). Lateral and angular variations of body segments and body center of mass were analysed, and their evolution over the learning period was compared to the changes in dimensional variables capturing the structure of the movement itself (embedding and correlation dimension, largest Lyapunov exponent). Results indicated that (i) learning occurred, (ii) was accompanied by persistence in the dimension of the movement, and (iii) by a reduction in chaotic (or stochastic) components. Compared to other results in the learning literature, these results suggest that dimensional changes over learning are task-specific.
\end{abstract}

(C) 2006 Elsevier Ireland Ltd. All rights reserved.

Keywords: Degrees of freedom; Balance control; Dimensionality; Learning

These last two decades or so, theories of motor learning have progressively incorporated tools from synergetics [11] and dynamical system theories [2] to account in the same framework for persistence and changes occurring during the acquisition of new sensori-motor skills. Instead of focusing on the processes operating within the central nervous system-or their information-processing psychological equivalents-the dynamical approach to motor learning emphases the self-organizing principles underlying movement stabilisation and destabilization over time, under the shaping influence of environmental, task, and body-related constraints. Since Bernstein's [5] original formulation of the changes in the biomechanical components accompanying the mastering of new skills-freezing, releasing, exploiting the number of degrees of freedom involved in the movement (e.g., [31])—several researchers have proposed new methods to solve the degrees of freedom problem, related to the selection, among a high number of potential configurations in kinematic (e.g., joint) space, of the appropriate variables involved in the control space [23]. Maintaining an upright stance is a classical example in which many degrees of freedom at the neuro-muscular or joint level are compressed into a lowdimensional space with only a few degrees of freedom, synergies (e.g., [19]), or coordination patterns [3,4].

\footnotetext{
* Corresponding author. Tel.: +33141744 470 .

E-mail address: fabrice.megrot@insep.fr (F. Mégrot).
}

Interesting for the present study are the attempts over the last decade to quantify the active (not biomechanical) degrees of freedom of the sensori-motor system, and their change over the course of learning, to capture this dimensionality. It is also generally assumed that the number of degrees of freedom of the control structure (active degrees of freedom) is considerably lower than the number of degrees of freedom of the controlled system [15].

Dimensional analyses have been applied to various skills such as finger oscillation movements [15], tremor [21], tracking movements [9] and upright bipedal posture [18,24]; see [23] for a recent review). In this literature, the estimated dimension provides a wide range of values, from low dimension (just above 1) to very high dimension (8-10). Interpretations of this wide range of values are not easy. During development or learning, dimension can decrease [12,16,33] or increase [22] according to the type of task. The direction of the change (increase or decrease) seems to be constrained by task properties. In many cases, it is not the active degrees of freedom per se that are modulated, but rather the geometrical structure of the underlying attractor, often in the direction of more structured and predictable patterns.

Dimensional analyses can be coupled with an analysis of the predictability of the system, revealing its organisation. Research on postural control has very rarely used this type of analysis (see [7], for an exception). The measure of the largest Lyapunov 
exponent consists in the evaluation of the sensitivity to the initial conditions of a time series (e.g., [26]). It is a reliable indicator of the organization of the attractor in its phase space, and thus an interesting addition to the dimensional analysis. A complex system can for instance be highly organized and a simple system can on the contrary be chaotic. Learning a new movement can consist in a change in the dimension of the intrinsic dynamics of the movement (from complex to simple or from simple to complex) or/and a change in the structure of the movement itself. Therefore, dimensional and predictability analyses appear complementary. The first one is more quantitative (number of active degrees of freedom) while the second one is more qualitative (modification of the structure of the attractor in phase). The aim of the present study is to evidence the quantitative (dimension) and qualitative (largest Lyapunov exponent) changes in the body organization during learning to maintain an unstable balance.

Participants $(N=6)$ were asked to maintain their balance on a stabilometer (Lafayette Instrument, model 16020). The $60 \mathrm{~cm} \times 40 \mathrm{~cm}$ platform had its axis of rotation $20 \mathrm{~cm}$ above the feet level, and its maximal angular rotation was $\pm 16^{\circ}$ with regard to the horizontal axis. Participants stood on the platform in a comfortable Romberg position (side by side), with the shoulders perpendicular to its axis of rotation, and the arms in the back (see [18]). They were instructed to minimize the movement of the platform during a trial and do their best to avoid contact of its edges with the ground surface. Each participant received 15 experimental trials every day during six consecutive days of practice (total of 90 trials). Trial duration was $40 \mathrm{~s}$ (see Fig. 1).

The motion of body segments was recorded in three dimensions with a six-camera VICON 512 infra-red motion analysis system, at a sampling rate of $120 \mathrm{~Hz}$. Fifteen passive reflecting markers were positioned on various parts of the body (left and right shoulders, sternum, left and right hips, left and right thighs, left and right knees, left and right tibias, left and right ankles, and left and right toes), and were later used to calculate the position and motion of the global center of mass $\left(\mathrm{Cm}_{\mathrm{g}}\right)$, as well as of local centers of mass for the head $\left(\mathrm{Cm}_{h}\right)$, the trunk $\left(\mathrm{Cm}_{t}\right)$, and the left leg $\left(\mathrm{Cm}_{1}\right)$. Position data were filtered using a 2nd order Butterworth filter with a cut off frequency of $4 \mathrm{~Hz}$. All centers

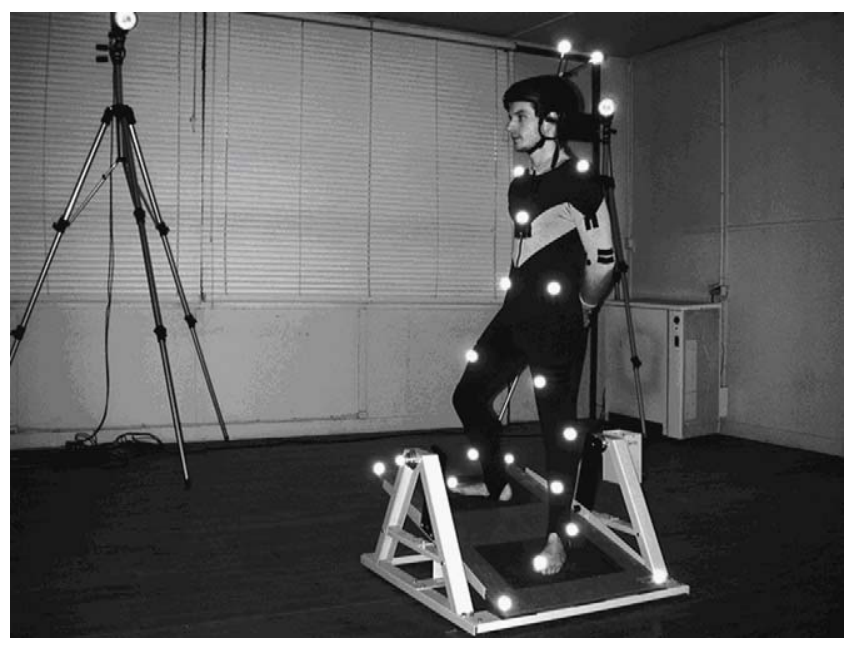

Fig. 1. Experimental apparatus. of mass were computed using Chandler et al.'s biomechanical model [6].

In each trial, phase space reconstruction was applied at each of these centers of mass and three variables were computed for local (head, trunk and leg) and global components: the embedding dimension (ED); the largest Lyapunov exponent $(\lambda)$ and the correlation dimension (CD).

Time series were analysed following Kantz and Schreiber's "Nonlinear Time Series Analysis" [14]. The procedure involved (i) the attractor reconstruction from the time series and the characterization of the chaotic dynamics by means of (ii) maximum Lyapunov exponent and (iii) correlation dimension. For (i), the matrix is characterized by two key parameters: The embedding dimension (ED) and the delay time $\tau$. The embedding dimension is the minimum dimension for which the reconstructed attractor can be considered completely unfolded with no overlap in the reconstructed trajectories. If the chosen dimension is lower than $\mathrm{ED}$, the attractor is not completely unfolded and the underlying dynamics cannot be investigated.

The algorithm used for the computation of ED was the 'Global False Nearest Neighbors' algorithm [1]. The delay time $\tau$ represents a measure that quantifies the reconstruction expansion from the identity line of the embedding space. In our study, $\tau$ was chosen using the first minimum point in the time delayed mutual information $[8,14,32]$. This technique measures the amount of information shared between two measurements $a$ and $b$. When the amount of information learned from a about $\mathrm{b}$ is at a minimum, the two time points are taken to be sufficiently independent [32].

For (ii), a trajectory is chaotic if there is at least one positive exponent. The value of this exponent, called the largest Lyapunov exponent $(\lambda)$ gives a measure of the divergence rate of infinitesimally close trajectories and of the unpredictability of the system. It thus gives a good characterization of the system's underlying dynamics. The Rosenstein-Kantz's method $[13,27,28]$ was used to compute $\lambda$ from the time series. This method measures in the reconstructed attractor the average divergence of two close trajectories in the amount of time considered.For (iii), the Grassberger-Procaccia algorithm [10] was used to determine the correlation dimension. The correlation dimension is an estimate of the least number of independent variables needed to characterize the system (given sufficient fine scale resolution). With each pass through the data, a new data point is taken, and a hyperdimension sphere of embedding dimension ED and radius $r$ is centred on that point. The fraction of subsequent data points in the record within that sphere $[C(R)]$ is then calculated for various values of $r$ (length scale), and a plot of $\log C(R)$ versus $\log r$ is performed for a range of embedding dimensions (for an embedding dimension of 2 in this case). The slope of this relation is the correlation dimension. These slopes were plotted against $r$ to identify values of the correlation dimension that were independent of both $r$ and ED. A correlation coefficient of 0.95 between the $\log [C(R)]-\log (R)$ plot and the linear regression was used as a criterion to exclude unreliable estimates of CD.

In order to test for spurious effects, the method of surrogate data was used [29]. Data were transformed in the frequency 
domain using a Fast Fourier Transform (FFT). Then, the complex components were multiplied by random phases uniformly distributed between 0 and $2 \pi$. The data were then transformed back in the temporal domain by computing an inverse FFT. The new time series now contains random numbers with the prescribed spectrum. This classical method, in addition to test for the artificial presence of chaos, allows to verify that the statistical effects obtained do exist and are not the consequence of the way $\mathrm{ED}, \lambda$, and $\mathrm{CD}$ are computed [14].

In addition to these phase space variables, kinematic variables were computed in order to assess the changes in biomechanical degrees of freedom during the 6 days of practice, i.e., (iv) the standard deviation of lateral displacement at each level, used to assess the relative stabilization of the segments and of the center of mass in that direction (lateral variability or LV); mean standard deviations of angular displacements of the platform, the hip, or the head with respect to the horizontal axis, or angular variation $A V_{p}, \mathrm{AV}_{\text {hip }}, \mathrm{AV}_{\text {head }}$. $\mathrm{AV}_{\mathrm{p}}$ were used as indexes of the participants' performance over the 6 days of practice, and $A V_{\text {head }}$ and $A V_{\text {hip }}$ provided information about angular segmental stabilization in the external space.

ED, LV and $\lambda$ data were submitted to a Day (1 to 6$) \times$ Levels (head, Cm, trunk, leg) MANOVA, followed by two-ways repeated measure ANOVAs on each of these variables. Due to the criterion used to exclude unreliable estimates values $(r \geq 0.95)$, the number of CD values differed between conditwo-ways ANOVAs.

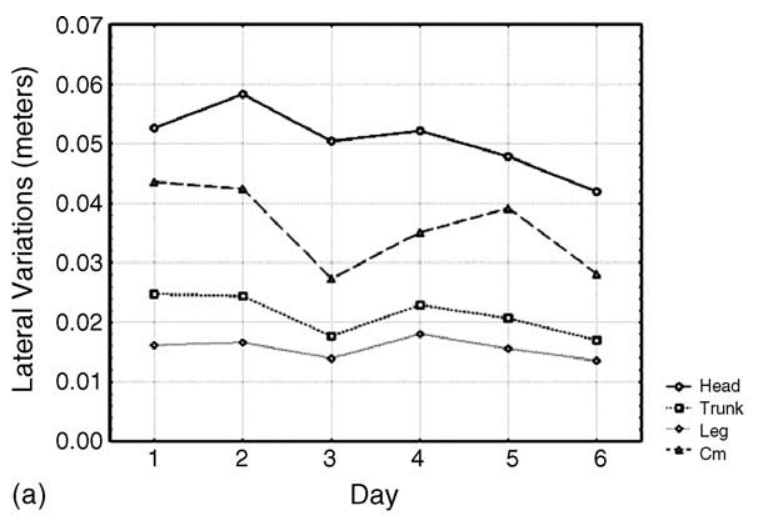
tions (see below). All AV measures were analysed in separate

Due to the conservative criterion used to exclude unreliable fits, $54 \%$ of the $\mathrm{CD}$ data were kept for analysis. The general MANOVA indicated a significant effect of Day, $R(15$, $5891)=15.36 p<.05$, and Level, $R(9,5193)=276.29 p<.05$. The interaction between these two factors was also significant, $R(15,6340)=2.03 p<.05$. We now proceed with univariate analyses for each of the dependent variables.

ED was 2 in each trial of each condition. The Day $\times$ Level ANOVA yielded no effect of Day, $F(5,2136)=1.00$, n.s., or level, $F(3,2136)=1.00$, n.s. This suggests that the geometry of the underlying dynamics was similar between levels and over practice, and did not exceed a limit cycle dynamics.

$\lambda$ had a mean of 0.12 (S.D. $=0.06$ ) across levels, conditions and trials, suggesting a modest divergence in the behavior of the postural system. Important differences, however, were found between levels and conditions. The Day $\times$ Level ANOVA indicated a main effect for Day, $F(5,2136)=36.42, p<.05$, the lateral movements of the body being more and more predictable as learning progressed. We can extract from Fig. 2c that Day-2, Day-3 and Day-4 were not different from each other (Newman-Keuls $p<.05$ ), indicating a non-linear decrease in divergence over the learning process.

There was also a main effect for the Level factor, $F(3$, $2136)=515.97, p<.05$. With one exception $\left(\mathrm{Cm}_{\mathrm{g}}\right.$ versus $\left.\mathrm{Cm}_{\mathrm{t}}\right)$, all levels significantly differed from each other (Newman-Keuls $p<.05)$. A proximo-distal gradient was found to exist, with lower values for the proximal components $\left(\mathrm{Cm}_{\mathrm{g}}, \mathrm{Cm}_{\mathrm{t}}\right)$ and higher values for the distal components $\left(\mathrm{Cm}_{\mathrm{h}}, \mathrm{Cm}_{1}\right)$, as
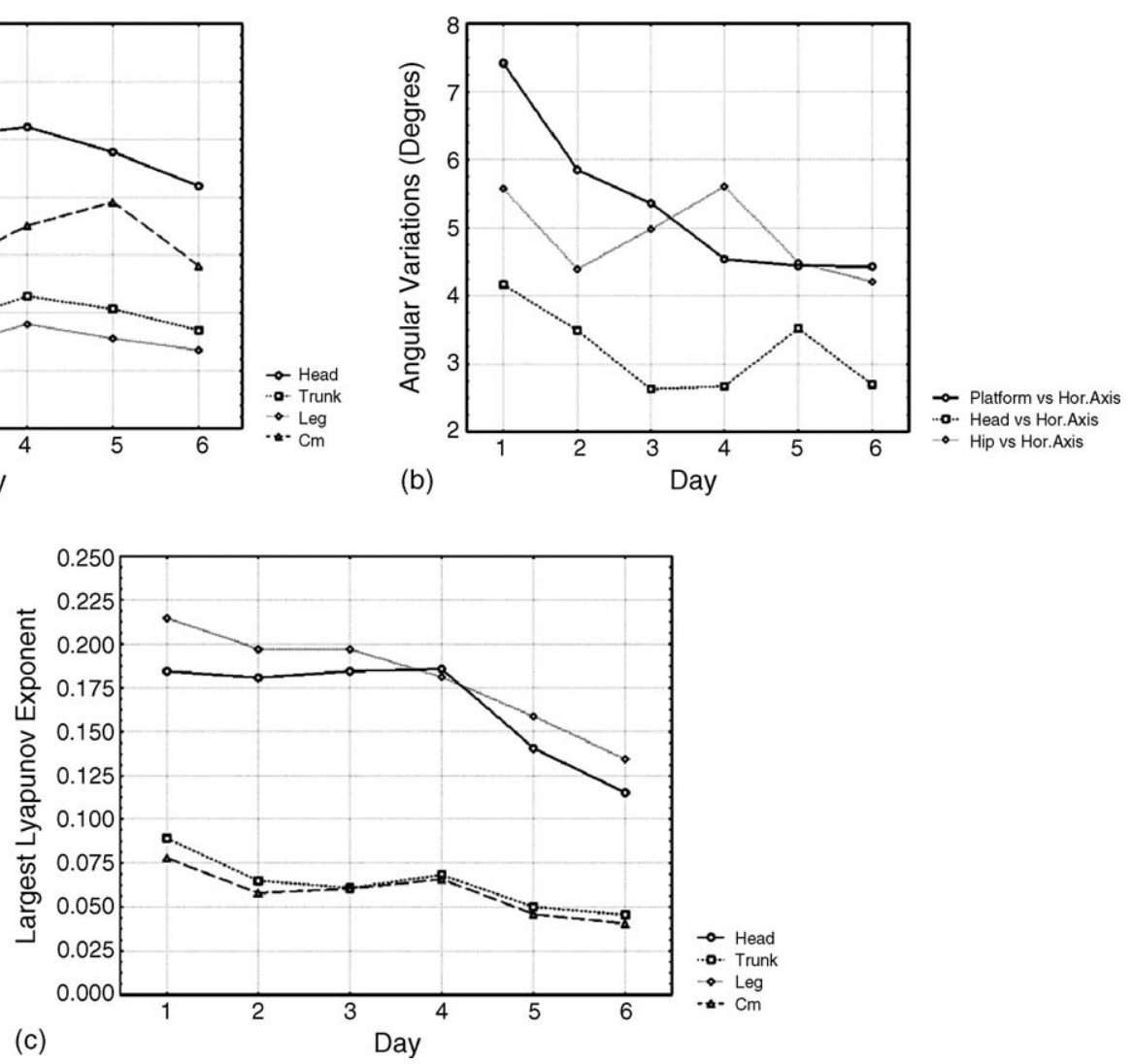

Fig. 2. (a) Lateral variability of head, trunk, leg, and global CM during the 6 days of practice. (b) Angular variability between head, hip, platform, and the horizontal axis during the 6 days of practice. (c) Largest Lyapunov exponent values of global (CM) and local (head, trunk, leg) centers of mass during the 6 days of practice. 
observed in Fig. 2c (Newman-Keuls $p<.05$ ). The interaction between the two factors was also significant, $F(15$, $2136)=2.68, p<.05$, and indicated a differential change in $\lambda$ over time, with the movement of the head being more predictable as learning progressed than movement of the other segments.

To test for spurious effects, the same analysis was performed on the surrogate values of $\lambda$. Values of surrogate $\lambda$ increased toward unpredictability, with a mean of 2.32 (S.D. $=0.45$ ). The surrogate values were about 20 times higher than the original values, $t(2159)=30.61, p<.05)$. In addition, The Day $\times$ Level ANOVA did not show any significant effect for Day, $F(5$, $2136)=0.071$, Level, $F(3,2136)=0.12$, or Day $\times$ Level interaction, $F(15,2136)=1.13, p>.05$, suggesting a rather erratic change in surrogate $\lambda$ between conditions. These results argue for the adequateness of the dimensional analysis to assess the (differential) presence of chaos (or even stochastic components) in the data and their decrease as learning progresses.

CD had a mean value of 0.73 (S.D. $=0.41$ ) across levels, days, and trials. There was no effect for day, $F(5,1173)=0.82$, n.s., and no effect for level, $F(3,1173)=1.80$, n.s. The Day $\times$ Level interaction was not significant, $F(5,1173)<1$. These results suggest that the underlying attractor was of low-dimensionality in each case.

CD was also computed on the surrogate data and compared with the original values. Similar to what was found for $\lambda$, a significant increase in dimension existed for the surrogate data, with means of 1.32 (S.D. $=0.27$ ). Original and surrogate values of CD differed, $t(1105)=21.87, p<.05$. The Day $\times$ Level ANOVA did not reach significance for Day, $F(5,1082)=2.81, p>.05$, for Level, $F(3,1024)<1$, or for the Day $\times$ Level interaction $F(15$, $1082)<1$.

Taken together, these results indicate that the observed values of $\lambda$ and $C D$ were attributable to the underlying dynamics of the postural system, and not to a potential artefact introduced by the dimensional analyses.

Mean lateral standard deviation was $3.5 \mathrm{~cm}($ S.D. $=0.68)$ for $\mathrm{Cm}_{\mathrm{g}}$, and was $5.05 \mathrm{~cm}$ (S.D. $\left.=1.28\right), 2.12 \mathrm{~cm}($ S.D. $=0.54)$, and $1.56 \mathrm{~cm}$ (S.D. $=0.32$ ), for $\mathrm{Cm}_{\mathrm{h}}, \mathrm{Cm}_{\mathrm{t}}$, and $\mathrm{Cm}_{1}$, respectively. Hence, the most immobilized segment in the environment was the leg, closely followed by the trunk, the global center of mass, and the head, exhibiting lateral movements of relative high amplitude. The Day $\times$ Level ANOVA performed on LV confirmed this result. A significant effect of Level was found for LV, $F(3,2136)=330.30, p<.05$, with all levels being significantly different from each other, post hoc Newman-Keuls $p<.05$. The Day $\times$ Level ANOVA indicated a main effect of practice, $F(5$, $2136)=14.18, p<.05$, showing a decrease in $\mathrm{LV}$ as learning progressed. Fig. 2a details these effects. The Day $\times$ Level interaction was also significant, $F(15,2136)=2.23, p<.05$, suggesting a differential decrease in lateral movement of the body over the 6 days of practice. As evidenced by post hoc Newman-Keuls tests $(p<.05)$, lateral movements of the head decreased more over time than movements of the other segments, with Day- 3 and Day- 6 being different from the other 4 days. These results evidenced a non-linear change over the learning session of body movements, with a singular decrease on Day-3 for all segments (see Fig. 2a).

Mean angular deviation with respect to the horizontal axis was $5.34^{\circ}($ S.D. $=2.29)$ for the platform, $3.20^{\circ}($ S.D. $=1.05)$ for the head, and $4.88^{\circ}$ (S.D. $=2.48$ ) for the hips. The head was the segment exhibiting the less amount of lateral rotation (see Fig. 2b). The Day $\times$ Level ANOVA performed on AV showed a significant effect of Level, $F(2,1602)=189.80, p<.05$, with all levels being significantly different from each other (post hoc Newman-Keuls $p<.05$ ). A main effect for Day was also found, $F(5,1602)=33.15, p<.05$, revealing less lateral rotation of the body at the end of the learning session than at the beginning. However, the significant Day $\times$ Level interaction, $F(10$, $1602)=10.02, p<.05$, completed the picture by showing that the changes in AV was not linear. Two important aspects of this interaction have to be noticed (Fig. 2b). First, lateral rotation decreased more for the head and the platform than for the hips. The decrease in platform rotation confirmed that participants did follow the instructions given by the experimenter (which was to minimize the movement of the platform) and learned the task over the 6 days of practice. Second, changes in AV were mostly noticeable during Day-1 and Day-6. As a matter of fact, Day-2, Day-3, Day-4, and Day-5 did not differ between each other, but were different from the other 2 days, Newman-Keuls $p<.05$.

Learning occurred. The results obtained in this study showed that participants improved their performance over the 6 days of practice (from $7.5^{\circ}$ the first day to $4.5^{\circ}$ the last day for mean angular variation of the platform). However, this improvement in performances was not accompanied by a variation in dimension. In other words, learning to maintain an unstable balance cannot be understood as a simplification of the performed movements. The similarity in the values of CD at global and local levels (between 0 and 1) suggests that the behavior of the postural system aims towards immobility. This low-dimensional dynamics certainly expresses the regulatory processes underlying balance control as well as the constraints acting upon the postural system. These results are not irrational if we consider that the unstable platform cannot be perfectly immobilized (dimension of 1), or sustain perfectly rhythmic movements (dimension of 2). The observed absence of dimensional variation over time runs counter other observations in the field of motor learning [23] and suggests that different levels of constraints (low, high) impose different organization of the movement during learning.

Although a similarity in dimension was observed between distal $\left(\mathrm{Cm}_{\mathrm{h}}, \mathrm{Cm}_{\mathrm{l}}\right)$ and proximal $\left(\mathrm{Cm}_{\mathrm{g}}, \mathrm{Cm}_{\mathrm{t}}\right)$ components of the postural system, the behavior of the proximal components $(\mathrm{Cm}$ and trunk) was found to become more predictable than the behavior of distal constituents (legs and head) as learning progressed. For instance, a minimization of $\lambda$ for $\mathrm{Cm}_{\mathrm{g}}$ was observed between Day-1 (mean of 0.077) and Day-6 (mean of 0.035). Although formally (i.e., mathematically), predictability implies a null value of $\lambda$, in biological movements with motion-capture related noise and biomechanical modelling, the significant decrease in $\lambda$ over time suggests a route towards predictability. This, in turns, indicates that participants modified the structure of their attractor to improve their performance. 
The literature in postural control is prolix with respect to the stabilizing role of the head in the regulation of stance (e.g., $[17,20,25])$. The theory of head stabilization indeed suggests that the position of a stabilized segment (in the external environment) can provide a-perceptual or physical-reference frame for the organization of the movement. This was not the case in our study. The head exhibited a highly divergent behavior (mean $\lambda$ of 0.165 over the 6 days), and its lateral variations were important (mean of 0.05 meters over the 6 days), creating both mechanical and perceptual (e.g., optical) changes. However, the continuous reduction over time of the angular displacement of the head, associated with the decrease of $\lambda$ over the last 2 days suggests an increasing role of perceptual components during learning. The angular rotation of the head during standing creates a rotary optical flow (e.g., [30]) which in turns can be used to regulate balance. Because the distance between the head and the surroundings remained more or less invariant in this task, the progressive minimization of head angular rotation over time reduces the rotary optical component, which in turns, may contribute to stabilize posture. We did not manipulate the available visual information in this study (but see [18]), and thus cannot conclude about its role in stabilizing posture during learning. The forcing role of optical flow in modulating the dynamic variables analysed in this contribution (dimension and predictability) needs to be assessed and is the focus of our current research.

\section{Acknowledgments}

The research reported in this article was supported by the French Ministère de la Recherche (Fond National pour la Science, Cognitique A118, and Institut Universitaire de France), and by Enactive Interfaces, a network of excellence (IST contract \#002114) of the Commission of the European Community. We would like to thank Mr. Albert Gaudin (Biometrics France) for providing the VICON motion analysis system, and Sofiane Ramdani for helpful comments on the manuscript.

\section{References}

[1] H.D.I. Abarbanel, M.B. Kennel, Local false nearest neighbours and dynamical dimensions from observed chaotic data, Phys. Rev. 47 (1993) 3057.

[2] F.D. Abraham, C.D. Shaw, Dynamics. The geometry of behavior. I. Periodic behavior, Aerial Press, Santa Cruz, CA, 1982.

[3] B.G. Bardy, L. Marin, T.A. Stoffregen, R.J. Bootsma, Postural coordination modes considered as emergent phenomena, J. Exp. Psychol.: Hum. Percept. Perform. 25 (1999) 1284-1301.

[4] B.G. Bardy, O. Oullier, R.J. Bootsma, T.A. Stoffregen, The dynamics of human postural transitions, J. Exp. Psychol.: Hum. Percept. Perform. 28 (2002) 499-514.

[5] N. Bernstein, The Co-ordination and Regulation of Movement, Pergamon, London, 1967.

[6] R.F. Chandler, C.E. Clauser, J.T. McConvile, H.M. Reynolds, J.W Young. Investigation of inertial properties of the human body (AMRLTR-137). Aerospace Medical Research Laboratories, Aerospace Medical Division (NTIS No AD-AO16 485). Wright-Patterson Air force Base, OH, USA, 1975
[7] J.J. Collins, C.J. De Luca, Random walking during quiet standing, Phys. Rev. Lett. 73 (1994) 764-767.

[8] A.M. Fraser, H.L. Swinney, Independent coordinates for strange attractors from mutual information, Phys. Rev. A 33 (1986) 11341140.

[9] R.E. Ganz, W.H. Ehrenstein, C.R. Cavonius, Dynamic complexity of visuo-motor coordination: an extension of Bernstein's conception of the degrees-of-freedom problem, Biol. Cybern. 75 (1996) 381-387.

[10] P. Grassberger, I. Procaccia, Measuring the strangeness of strange attractors, Physica D 9 (1983) 189-208.

[11] H. Haken, Synergetics: An Introduction, Springer, Berlin, 1983.

[12] H. Haken, Principles of Brain Functioning: A Synergetic Approach to Brain Activity, Behavior and Cognition, Springer, Berlin, 1996.

[13] H. Kantz, A robust method to estimate the maximal lyapunov exponent of a time series, Phys. Lett. 185 (1994) 77.

[14] H. Kantz, T. Schreiber, Nonlinear Time Series Analysis, Cambridge University Press, Cambridge, 1997.

[15] B.A. Kay, The dimensionality of movement trajectories and the degrees of freedom problem: a tutorial, Hum. Mov. Sci. 7 (1988) 343364.

[16] P.N. Kugler, M.T. Turvey, Information, Natural Law, and the Selfassembly of Rhythmic Movement: Theoretical and Experimental Investigations, Lawrence Erlbaum, Hilsdale, NJ, 1987.

[17] J. Massion, Movement, posture, and equilibrium: Interaction and coordination, Prog. Neurobiol. 38 (1992) 35-56.

[18] F. Mégrot, B.G. Bardy, G. Dietrich, Dimensionality and the dynamics of human unstable equilibrium, J. Mot. Behav. 34 (2002) 323-326.

[19] L.M. Nashner, G. Mac Collum, The organization of postural movements: a formal basis and experimental synthesis, Behav. Brain Sci. 8 (1985) $135-172$.

[20] L.M. Nashner, C.L. Shupert, F.B. Horak, F.O. Black, Organization of posture control: an analysis of sensory and mechanical constraints, Prog. Brain Res. 80 (1989) 411-418.

[21] K.M. Newell, F. Gao, R.L. Sprague, The dynamics of finger tremor in tardive dyskinesia, Chaos 5 (1995) 43-47.

[22] K.M. Newell, Y.T. Liu, G. Mayer-Kress, The sequential structure of movement outcome in learning a discrete timing task, J. Mot. Behav. 29 (1997) 366-382.

[23] K.M. Newell, D.E. Vaillancourt, Dimensional change in motor learning, Hum. Mov. Sci. 20 (2001) 695-715.

[24] K.M. Newell, R.E.A. van Emmerik, D. Lee, R.L. Sprague, On postural stability and variability, Gait Posture 4 (1993) 225-230.

[25] T. Pozzo, A. Berthoz, L. Lefort, Head stabilization during various locomotor tasks in Humans. I: Normal Subjects, Exp. Brain Res. 82 (1990) 97-106.

[26] A. Prigogine, I. Stengers, La nouvelle alliance, Gallimard, Paris, 1979.

[27] M.T. Rosenstein, J.J. Collins, C.J. De Luca, A practical method for calculating largest Lyapunov exponents from small data sets, Physica D 65 (1993) 117-134.

[28] M.T. Rosenstein, J.J. Collins, C.J. De Luca, Reconstruction expansion as a geometry-based framework for choosing proper delay times, Physica D 73 (1994) 82-98.

[29] J. Theiler, S. Eubank, A. Longtin, B. Galdrikian, J.D. Farmer, Testing for nonlinearity in time series: the method of surrogate data, Physica D 58 (1992) 77.

[30] W.N.J.C. Van Asten, C.C.A.M. Gielen, J.J.D. Denier van der Gon, Postural movements induced by rotation of visual scenes, J. Opt. Soc. Am. A5 (1988) 1781-1789.

[31] B. Vereijken, R.E.A. van Emmerik, H.T.A. Whiting, K.M. Newell, Free(z)ing degrees of freedom in skill acquisition, J. Mot. Behav. 24 (1992) 133-142.

[32] H.D.I. Abarbanel, Analysis of chaotic observed data, Springer, New York, 1996.

[33] S. Mitra, P.G. Amazeen, M.T. Turvey, Intermediate motor learning as decreasing active (dynamical) degrees of freedom, Hum. Mov. Sci. 17 (1998) 17-66. 